\title{
Effect Of Heating Treatment and Mixture On Optical Properties Of Coating Materials Used In Gravitational-Wave Detectors
}

\author{
${ }^{1}$ Alex Amato, a) ${ }^{2}$ Silvana Terreni, ${ }^{1}$ Massimo Granata, ${ }^{1}$ Christophe Michel, ${ }^{1}$ Laurent Pinard, ${ }^{3}$ Gianluca Gemme, \\ ${ }^{23}$ Maurizio Canepa, and ${ }^{4}$ Gianpietro Cagnoli \\ ${ }^{1}$ Laboratoire des Matériaux Avancés, CNRS/IN2P3, F-69622 Villeurbanne, France. \\ ${ }^{2}$ OPTMATLAB, Dipartimento di Fisica, Università di Genova, Via Dodecaneso 33, 16146 Genova, \\ Italy. \\ ${ }^{3}$ INFN, Sezione di Genova, Via Dodecaneso 33, 16146 Genova, Italy. and \\ ${ }^{4}$ University of Lyon, Université Claude Bernard Lyon 1, CNRS, Institut Lumière Matière, F-69622, \\ VILLEURBANNE, France.
}

(Dated: 1 October 2019)

The interferometer mirrors of Gravitational-Wave Detectors (GWD) are Bragg reflectors made of alternate amorphous silica $\left(\mathrm{SiO}_{2}\right)$ and titania-doped tantala $\left(\mathrm{TiO}_{2}: \mathrm{Ta}_{2} \mathrm{O}_{5}\right)$ layers as low- and high-refractive index material, respectively. A thermal treatment is usually performed to reduce both mechanical losses and NIR optical absorptions of the coatings. We present a spectroscopic ellipsometry (SE) investigation of the effect of annealing and $\mathrm{Ti}$ :Ta mixing on $\mathrm{Ta}_{2} \mathrm{O}_{5}$ coatings deposited under conditions similar to those adopted for building up mirrors of GWDs. The broad-band analysis covers both the NIR and the fundamental absorption threshold region. The data show an evident annealing-induced reduction of the fundamental optical absorption broadening. Modelling the data through the Cody-Lorentz formula confirms that NIR absorption are below the SE sensitivity and shows a notable annealing-induced reduction of so-called Urbach tails. Titania-doping of tantala slightly reduces the Urbach energy. After the heating treatment the resulting Urbach energy of the doped material is lower than the one of annealed pure tantala. The observed reduction of Urbach tails is important because it parallels the reduction of so-called internal friction observed in mechanical measurements. So that SE emerges as a convenient tool for an agile diagnostic of both optical and mechanical quality of amorphous oxide coatings.

\section{INTRODUCTION}

VIRGO and LIGO Gravitational-wave detectors (GWD) are giant interferometers that use multi-layer Bragg reflectors deposited on large, massive fused silica substrates which play the role of gravitational-field test-masses ${ }^{1}$. The Bragg reflectors are made of a stack of high-quality, amorphous oxide layers. Alternate layers of silica $\left(\mathrm{SiO}_{2}\right.$, the "low-index" material) and so called titania-doped tantala $\left(\mathrm{TiO}_{2}: \mathrm{Ta}_{2} \mathrm{O}_{5}\right.$, the "highindex" material) have been adopted by the Advanced LIGO $^{2}$ (aLIGO) and Advanced Virgo ${ }^{3}$ (AdV) detectors. The layers are deposited by a specifically-designed ion beam sputtering (IBS) apparatus, the so-called Grand Coater at the LMA (Lyon, France). Accurate protocols of deposition and post-deposition thermal procedures (annealing) allow to produce uniform and homogeneous coatings endowed with extremely low optical absorption (few ppm) at the laser operational wavelength (1064 nm), and low internal friction. The Bragg reflectors are crucial elements of GWDs as the coating thermal noise actually limits the detection sensitivity ${ }^{4}$. Therefore, although presently adopted materials and geometries already warrant great performances which contributed to the recent detection of a number of GW events ${ }^{5}$, Virgo and LIGO groups are involved in a difficult task for improving further the optical and mechanical properties of coatings.

a)Electronic mail: a.amato@lma.in2p3.fr
Optical methods clearly play an important role in this search. As a matter of facts, careful characterizations of the refractive index and extinction coefficient at the interferometer operational wavelength are necessary ${ }^{6,7}$ as well as a determination of the actual thickness of layers. Spectroscopic Ellipsometry (SE) measurements can provide accurate information in this respect as demonstrated by several works ${ }^{8,9}$. This paper however wants to focus on another relevant aspect. There is a certain consensus about the fact that the high-refractive index material is the main source of thermal noise in the mirror coating. "Doping" $\mathrm{Ta}_{2} \mathrm{O}_{5}$ with $\mathrm{TiO}_{2}$ was the solution found to decrease the mirror thermal noise ${ }^{4,10,11}$. Such improvements have been obtained somewhat "empirically" and it is now believed that a better understanding of the intimate relation between the structural properties of amorphous oxides and internal friction effects ${ }^{12}$ can be functional to the optimization of "doping" and annealing procedures.

According to the fluctuation-dissipation theorem ${ }^{13}$ thermal noise arises from fluctuations of atomic positions in substrate and coating structure ${ }^{14}$, which allows several equilibrium configurations.

The width of so-called Urbach tails ${ }^{15}$ at the fundamental absorption edge is controlled by the mean square displacement of atoms from their equilibrium positions, where the contribution of structural disorder (dominant in amorphous materials) adds quadratically to the contribution of thermal disorder ${ }^{16}$.

Several recent theoretical (computational) studies on 
amorphous semiconductors ${ }^{17,18}$ highlighted localized tail states in electronic DOS, near in energy to the extended states, which reflect the presence of a spatial correlation between defects. These studies showed that amorphous semiconductors may display, especially after suitable thermal treatments, peculiar and interesting forms of short- and medium-range atomic organization ${ }^{19,20}$. In a-Si there are for instance connected sub-networks of short bonds which induce Urbach states near the valence band edge. More interesting, if such medium-range atomic organization is artificially destroyed in the model, the Urbach tail broadening, usually quantified by the socalled Urbach energy, increases ${ }^{19}$. So in a-Si atomic self organization of the structure promoted by annealing reduces the Urbach energy. On the base of these works on a-semiconductors, we can suppose that annealing procedure (and perhaps "doping") can favour the emerging of not crystalline, medium-range atomic organization that relieves the strain in the amorphous network and induces a reduction of Urbach broadening. The resulting structure presents therefore lower Urbach energies and lower internal friction, two different properties likely connected via structure ${ }^{21}$. In this perspective, an accurate characterization of the fundamental absorption edge, in particular of Urbach tails, of amorphous semiconductors can be envisaged to offer important insight in the understanding of the relation between electronic and atomic structure with internal friction, a parameter which quantifies the mechanical loss ${ }^{22}$

We used broad-band SE to investigate optical properties and thickness of high-index coating materials films $\left(\mathrm{Ta}_{2} \mathrm{O}_{5}\right.$ and $\left.\mathrm{TiO}_{2}: \mathrm{Ta}_{2} \mathrm{O}_{5}\right)$ before and after the annealing at $500^{\circ} \mathrm{C}$, with special focus to the absorption edge region. The well-known Cody-Lorentz (CL) model, which explicitly considers the Urbach broadening, is adopted to model the data. Our analysis unequivocally shows that annealing, primarily, and "doping" lead to a notable reduction of the Urbach tails extension.

Finally, we note that Bragg mirrors with low thermal noise are important also for other precision experiments using high-finesse optical cavities, such as laser frequency stabilization cavities ${ }^{23}$, opto-mechanical resonators ${ }^{24}$ and atomic clocks ${ }^{25}$.

\section{EXPERIMENT}

Samples are single layers of $\mathrm{Ta}_{2} \mathrm{O}_{5}$ and $\mathrm{TiO}_{2}: \mathrm{Ta}_{2} \mathrm{O}_{5}$ deposited by ion beam sputtering (IBS) at the Laboratoire des Matériaux Avancés (Lyon, France) using a large custom-developed coating chamber, so-called Grand Coater (GC). GC has been built specifically to coat the mirrors for GWD with very high thickness uniformity over a large surface ${ }^{26}$.

The doped material has been obtained by co-sputtering in $\mathrm{O}_{2}$ atmosphere at $10^{-4}$ mbar, in order to obtain $\mathrm{Ta}_{2} \mathrm{O}_{5}$ and $\mathrm{TiO}_{2}$ particles reaching the substrate during the deposition. The ratio of Ti to Ta of the doped material has been measured through Rutherford backscattering spectrometry (RBS), taken on witness samples. In particular, the percentage atomic concentrations obtained by $\mathrm{RBS}$ are $\mathrm{Ta}=22.2 \pm 0.7, \mathrm{Ti}=6 \pm 0.6, \mathrm{O}=69 \pm 7$ and $\mathrm{Ar}=4 \pm 2$, thus giving a Ti to Ta ratio of $0.27 \pm 0.04$ (the presence of argon in the coating is due to the deposition method). SE measurements in the reflection mode need a reflective substrate, and precautions to avoid backside reflections from the bottom of the substrate itself. In this respect, silicon wafers $1 \mathrm{~mm}$ thick, having 2" of diameter and one un-polished surface, have been coated on the polished surface for optical characterization.

As part of the standard post-deposition process of mirrors for GWD, all the coated samples underwent in-air annealing at $500^{\circ} \mathrm{C}$ over 10 hours. This is necessary to decrease both the internal mechanical stress and the optical absorption of the coatings ${ }^{27,28}$. All the measurements have been carried out on as-deposited and annealed samples. For sake of clarity, if not specified otherwise, the data refer to the sample after the annealing.

The optical properties, as well as the coating thickness, have been determined by spectroscopic ellipsometry $(\mathrm{SE})^{29}$ using two different instruments: a rotating compensator ellipsometer (J. A. Woollam M-2000), operating in the $245-1700 \mathrm{~nm}$ wavelength range probing 674 points, and a VASE ellipsometer (J.A. Woollam), operating in the 190-1100 $\mathrm{nm}$ range probing 910 points. The combination of the two instruments allowed thus obtaining information related to a wavelength range extended from 190 to $1700 \mathrm{~nm}(0.7-6.5 \mathrm{eV})$. Data have been acquired at three different angles $\left(55^{\circ}, 60^{\circ}\right.$, $\left.65^{\circ}\right)$. The analysis was done independently for VASE and M-2000 sets of data. The two ellipsometers gave comparable results in the overlapping spectral region. For the sake of clarity, only the data obtained with the VASE will be shown.

Following previous works $^{8,9}$, SE data were analysed through a tree-layer system (ambient/coating/substrate). On the top of the coating a Bruggeman effective medium approximation (EMA) layer, which accounts for roughness, has been modelled. We note that the roughness obtained on these coatings is less than $1 \mathrm{~nm}$, in agreement with atomic force microscopy (AFM) measurements of root mean square (RMS) roughness on this kind of samples ${ }^{9}$, and therefore does not affect the final results.

The optical properties of bare substrate have been pre-determined by dedicated measurements, as done in ref. ${ }^{8}$. The optical parameters and the thickness of the coating have been then determined by fitting the $(\Psi, \Delta)$ data. The complex dielectric function $\varepsilon$ of the absorbing coatings have been modelled in the whole energy region by the well-known Cody-Lorentz (CL) model ${ }^{30}$ and the so-called Herzinger-Johs parametrized semiconductor (Psemi) model ${ }^{31}$ as done for instance in ref. ${ }^{27}$. The two models gave closely comparable results. For a critical discussion of the CL model, specially regarding the 

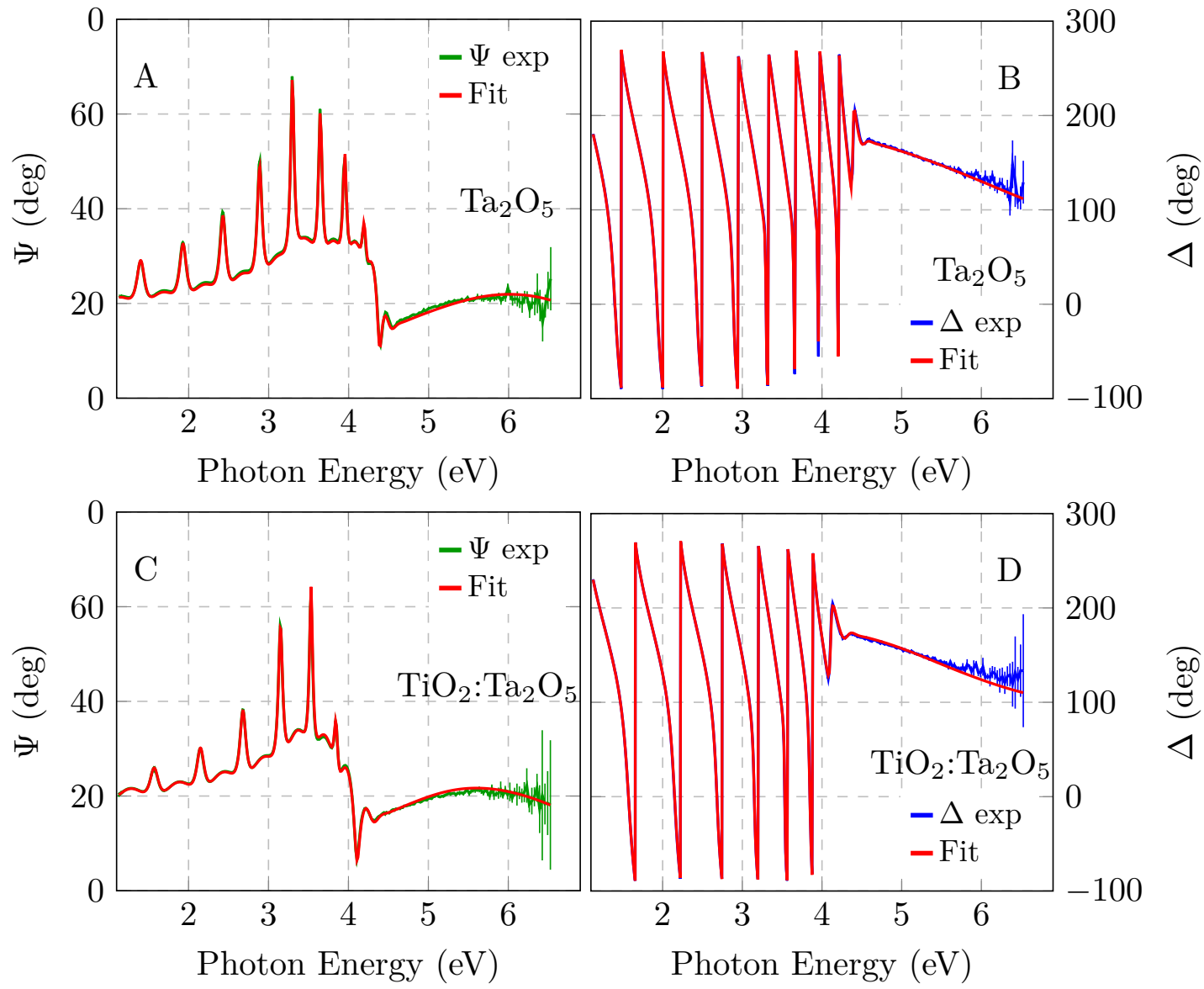

FIG. 1. Comparison between $\Psi$ (green), $\Delta$ (blue) data at $60^{\circ}$ obtained from the annealed samples and best fit (red). A), B) SE data of $\mathrm{Ta}_{2} \mathrm{O}_{5}$ coating measured with VASE ellipsometer and simulations obtained by CL model. C), D) SE data of $\mathrm{TiO}_{2}: \mathrm{Ta}_{2} \mathrm{O}_{5}$ coating measured with VASE ellipsometer and simulations obtained by CL model.

KK-consistency, we address to the classical article of Ferlauto et al. ${ }^{30}$ and more recent works ${ }^{32,33}$. In this respect it is worth noting that the coatings under study present extremely low NIR absorptions, out of the range detectable by SE measurements. As a matter of facts, regarding the NIR region, the CL and PSEMI models - applied to the extended wavelength range - provided results comparable to those obtained by application of the simplest Cauchy model to the transparent range. In the following, for brevity, we will report about the results obtained with the CL model, which includes explicitly the so-called Urbach correction to the fundamental edge broadening. The optical gap is also included as a fit parameter in the model. More information and rigorous definition of the CL model parameters, applied to this kind of samples, can be found in appendix A of ref. ${ }^{8}$.

\section{RESULTS}

In figure $1 \mathrm{~A}, \mathrm{~B}$ the data obtained from $\mathrm{Ta}_{2} \mathrm{O}_{5}$ coating after the annealing are shown, together with the best fit curves. For clarity, only the data at $60^{\circ}$ are shown. The data obtained from $\mathrm{TiO}_{2}: \mathrm{Ta}_{2} \mathrm{O}_{5}$ coating are presented in panels C,D. Common to the two sets of data, one can observe that the interference oscillations well evident in the NIR-Vis region fade when approaching the UV, highlighting the absorption threshold. This behaviour is perhaps more appreciable in $\Psi$ data, where one can guess the presence of absorption broadening (Urbach tail) inside the gap. The $(\Psi, \Delta)$ data show a degradation in the deeper UV region caused also by the strong absorption which reduces the signal to noise ratio.

A comparison of measurements taken on tantala samples before and after the annealing, shown in figure 2, emphasizes how the main difference induced by the heating treatment is just around the absorption threshold, in the Urbach tail region. The annealing seems not to affect the energy gap while after the annealing, there are oscillations appearing near the absorption threshold. This suggests that the absorption, related to the Urbach tails, is reduced.

In order to test the effect of the Urbach correction, we compared $(\Psi, \Delta)$ data of $\mathrm{Ta}_{2} \mathrm{O}_{5}$ coating before the annealing, which presents higher sub-gap absorptions, with simulations based on two CL models. In one model we 

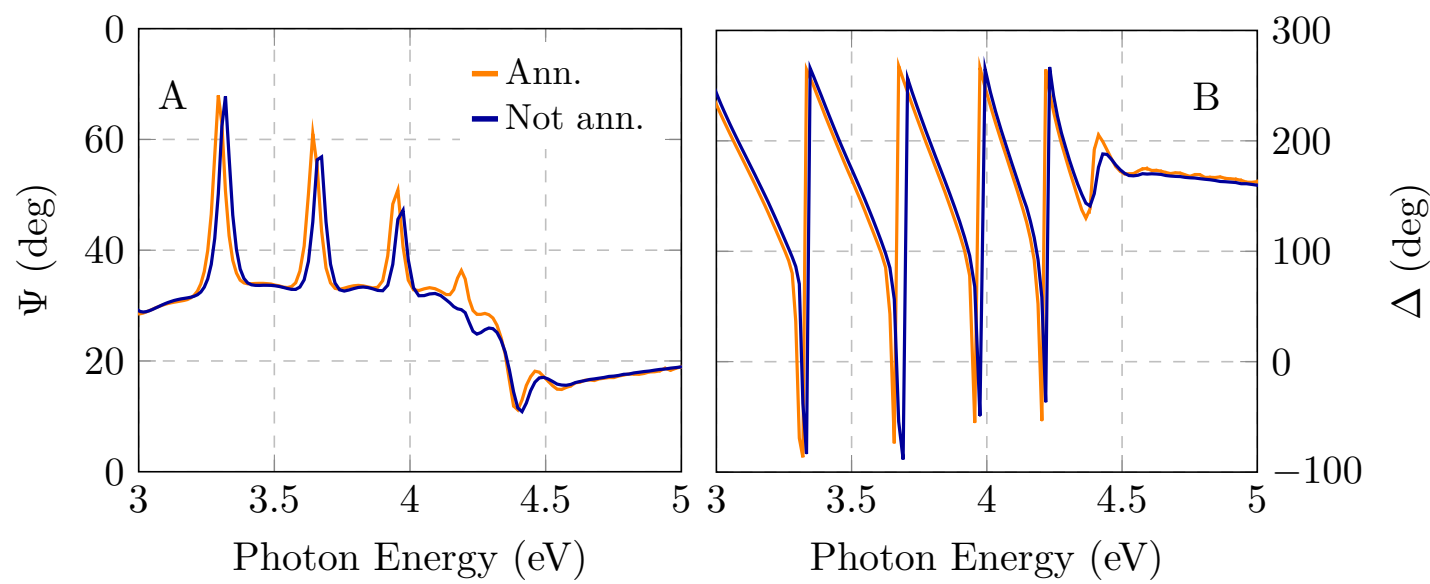

FIG. 2. A) $\Psi$ and B) $\Delta$ data at $60^{\circ}$ for $\mathrm{Ta}_{2} \mathrm{O}_{5}$ coating before (blue) and after (orange) the annealing.

TABLE I. Thickness and density of samples before and after the annealing.

\begin{tabular}{lcccc}
\hline \hline \multirow{2}{*}{ Coating } & \multicolumn{2}{c}{ Thickness $(\mathrm{nm})$} & \multicolumn{2}{c}{ Density $\left(\mathrm{g} / \mathrm{cm}^{3}\right)$} \\
& As deposited Annealed & As deposited & Annealed \\
$\mathrm{Ta}_{2} \mathrm{O}_{5}$ & $579 \pm 2$ & $592 \pm 2$ & $7.40 \pm 0.03$ & $7.33 \pm 0.06$ \\
$\mathrm{TiO}_{2}: \mathrm{Ta}_{2} \mathrm{O}_{5}$ & $500 \pm 2$ & $509 \pm 2$ & $6.87 \pm 0.06$ & $6.65 \pm 0.07$ \\
\hline \hline
\end{tabular}

excluded the Urbach tails; the fit (not shown) reproduced the $(\Psi, \Delta)$ behaviour with a mean squared error (MSE) of about 6.4. The main differences between the fit curves and the data were concentrated at the absorption edge, where the fits differed more than $10 \%$ from the data. The second CL model included the Urbach tails and the best fit reproduced the $(\Psi, \Delta)$ data with a MSE of about 4.0. The differences between fit and data at the absorption edge have been reduced under $5 \%$, demonstrating that the Urbach tails are needed in the model to better reproduce the data. The two models indeed gave comparable optical properties regarding the refractive index in the transparent region, the energy gap and thickness. The same analysis has been done also for $\mathrm{TiO}_{2}: \mathrm{Ta}_{2} \mathrm{O}_{5}$ and similar conclusions could be drawn. For this reason, the best fit curves shown in figure 1 and the following analysis refer to the CL model which includes the Urbach correction. Note that the analysis has been done at the three angles of incidence, and the fit curves reproduced the $(\Psi, \Delta)$ data with similar accuracy.

The analysis shows that the annealing increases the coating thickness of about $2 \%$. The coating thickness has been then used to estimate the density of the material. The thickness and the density of the samples are listed in table I. It is known that IBS technique provides dense coatings ${ }^{34}$. It is reasonable to assume that the annealing induces some relaxations of the coating structure, with a consequent increase of the coating thickness. The annealing slightly reduces the refractive index in the transparent region, as shown in figure $3, \mathrm{~A}-\mathrm{B}$ and in table
TABLE II. Refractive index at $1064 \mathrm{~nm}$, energy gap $E_{g}$ and Urbach energy $E_{U}$ of coatings before and after the annealing at $500^{\circ} \mathrm{C}$. The optical properties have been worked out using the CL model.

\begin{tabular}{lccc}
\hline \hline Coating & $n @ 1064 \mathrm{~nm}$ & $E_{g}(\mathrm{eV})$ & $E_{U}(\mathrm{meV})$ \\
$\mathrm{Ta}_{2} \mathrm{O}_{5}$ & $2.07 \pm 0.01$ & $4.1 \pm 0.2$ & $164 \pm 8$ \\
$\mathrm{Ta}_{2} \mathrm{O}_{5}$ ann. & $2.05 \pm 0.01$ & $4.0 \pm 0.1$ & $130 \pm 10$ \\
$\mathrm{TiO}_{2}: \mathrm{Ta}_{2} \mathrm{O}_{5}$ & $2.11 \pm 0.01$ & $3.6 \pm 0.1$ & $152 \pm 5$ \\
$\mathrm{TiO}_{2}: \mathrm{Ta}_{2} \mathrm{O}_{5}$ ann. & $2.09 \pm 0.01$ & $3.6 \pm 0.1$ & $108 \pm 5$ \\
\hline \hline
\end{tabular}

II. At $1064 \mathrm{~nm}$ the reduction is almost 1\%. Furthermore, by comparing refractive index in figure $3, \mathrm{~A}-\mathrm{B}$ it can be observed that the titania-doping increases the refractive index, in the NIR region, including $1064 \mathrm{~nm}$ (table II). The resulting refractive index of $\mathrm{Ta}_{2} \mathrm{O}_{5}$ is consistent with previous results ${ }^{35}$.

Note that the values of the refractive index obtained by the CL model in the NIR region are in agreement with those obtained by the Cauchy model applied to the transparent region. We have checked the thickness/index correlation which, for these relatively thick samples, turned out negligible.

In figure 3,C,D the extinction coefficients related to the best fit model are shown. Note that the models used to reproduce the $(\Psi, \Delta)$ data give values for the absorption coefficient at $1064 \mathrm{~nm}$ which are orders of magnitude below the intrinsic experimental sensitivity of SE. These films are endowed with ultra-low absorptions at $1064 \mathrm{~nm}$ which can be measured only by dedicated methods, for example based on photothermal deflection technique ${ }^{36}$. By comparing figures 3,C and 3,D, it can be observed that the titania-doping significantly reduces the energy gap $^{8}$, as reflected in the $E_{g}$ parameter (table II). This effect indeed can be observed by comparing directly the $(\Psi, \Delta)$ data in figure $1, \mathrm{~A}$ and $1, \mathrm{C}$. Note that the energy gap is poorly affected by the annealing, as shown in table II. On the other hand, there is a clear annealing-induced 

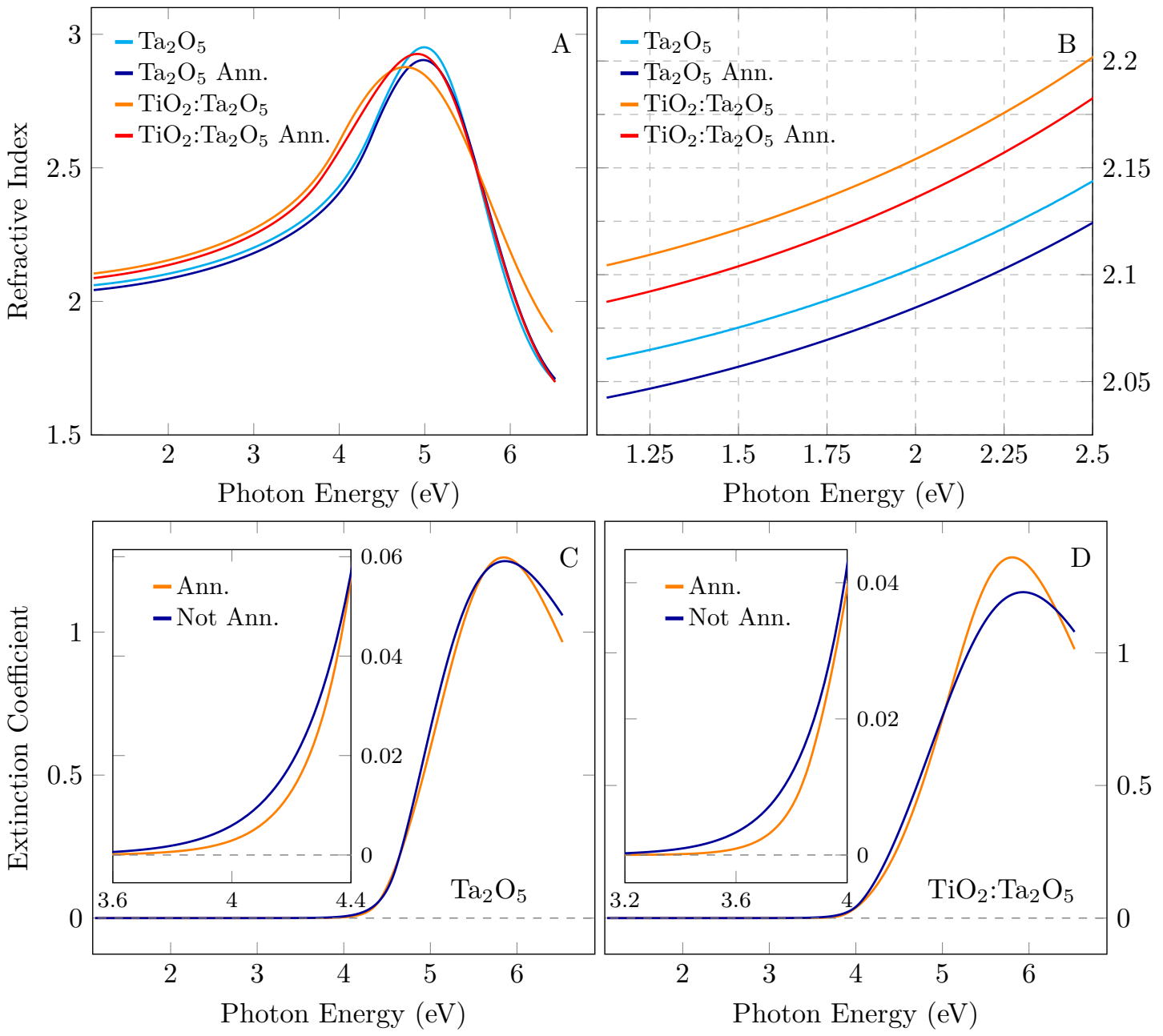

FIG. 3. Optical properties derived by fitting the CL model to the SE data. A) Refractive index of $\mathrm{Ta}_{2} \mathrm{O}_{5}$ coating before (light blue) and after (blue) the annealing compared with the refractive index of $\mathrm{TiO}_{2}: \mathrm{Ta}_{2} \mathrm{O}_{5}$ coating before (orange) and after (red) the annealing. B) Refractive index in IR-Vis region to better appreciate the differences in the transparent region. Extinction coefficient of C) $\mathrm{Ta}_{2} \mathrm{O}_{5}$ and D) $\mathrm{TiO}_{2}: \mathrm{Ta}_{2} \mathrm{O}_{5}$ coatings before (blue) and after (orange) the annealing. The insets zoom in the absorption threshold region.

change in the region of the absorption thresholds, which is better visible in the insets. Indeed, the extension of the Urbach tails is reduced after the annealing, as quantified by the Urbach energy parameter $E_{U}$ reported in table II. Note in the table that the Urbach energies for the doped sample are smaller than those of pure tantala. This result finds a graphic counterpart. Coming to a closer look to the doped tantala data in figure $1 \mathrm{C}, \mathrm{D}$, particularly $\Psi$ data, one can note a more pronounced damping of the peaked structures in the $3.5-4 \mathrm{eV}$ region. It is worth noting that the impact of the annealing on the extension of the Urbach tails is more evident than the one of the doping.

The reduction of the absorption broadening, associated to the Urbach tails, can be related to strain release and to structural organization of the material. In facts previous works showed that the annealing favours spatial correlation between defects, promotes short- and medium-range atomic organization and finally produces a reduction of structural inhomogeneity of the material ${ }^{19,20,37}$.

The effect of structural inhomogeneity of amorphous oxide coatings on internal friction has been already highlighted ${ }^{12,38}$.

\section{CONCLUSIONS}

Amorphous oxide coatings used as high-refractive index materials for Bragg reflectors in GWD have been analysed by SE, covering a broad energy region (190 $1700 \mathrm{~nm}$ ). The layers are $\mathrm{Ta}_{2} \mathrm{O}_{5}$ and $\mathrm{TiO}_{2}: \mathrm{Ta}_{2} \mathrm{O}_{5}$ grown by IBS on Si wafers, under the same conditions adopted in the realization of GWD mirrors. The data have been analysed using different models to simulate the dielectric function of the coating with focus on the Cody-Lorentz model which includes explicitly the gap energy and the 
so-called Urbach energy as free parameters. The analysis has been done on the samples before and after a post deposition heating treatment for $10 \mathrm{~h}$ at $500^{\circ} \mathrm{C}$. The annealing reduces the refractive index in the Vis-NIR region and increases the coating thickness, without significantly affecting the energy gap parameter. The titania-doping is responsible for a red-shift of the energy gap and an increase of the refractive index in the Vis-NIR region. A closer analysis of the absorption edge pointed out that the annealing significantly reduces the Urbach broadening. Titania-doping of tantala slightly reduces the Urbach energy. After the heating treatment the resulting Urbach energy of the doped material is lower than the one of annealed pure tantala. Considering recent theoretical works on amorphous oxides the reduction of the extension of Urbach broadening may indicate that the structure of the annealed, doped-tantala coating presents an atomic organization extending beyond the short range scale, which in turn may be the origin of the observed parallel reduction of mechanical losses. In this view an accurate characterization of the absorption edge of amorphous oxide coatings can be used to obtain indirect information on the amorphous structure of samples and to select promising candidates to be tested regarding the mechanical quality (e.g. the internal friction). Under this respect, the structure of the annealed titania-doped coating should be more organized and homogeneous than the as deposited un-doped one, suggesting an atomic organization of the amorphous oxide on a medium-long-range scale. In this view, the investigation of Urbach tails in the absorption edge of amorphous coatings emerges as a rapid and non-destructive method for analyse their structural homogeneity and mechanical quality.

${ }^{1}$ R. X. Adhikari, Rev. Mod. Phys. 86, 121-151 (2014).

${ }^{2}$ J. Aasi et al., Classical and Quantum Gravity 32, 074001. (2015).

${ }^{3} \mathrm{~F}$. Acernese et al., Classical and Quantum Gravity 32, 024001. (2015).

${ }^{4}$ G. M. Harry et al., Classical and Quantum Gravity 19, 897-917 (2002).

${ }^{5}$ The LIGO Scientific Collaboration and Virgo Collaboration, Living Rev. Relativ. 19, 1 (2016).

${ }^{6}$ L. Pinard et al., Appl. Optics 56, C11-C15 (2017).

${ }^{7}$ R. Flaminio et al., Classical and Quantum Gravity 27, 084030 (2010).

8 A. Amato et al., Journal of Physics: Materials 2, 035004 (2019).

${ }^{9}$ M. Prato et al., Thin Solid Films 519, 2877 - 2880 (2011), 5th International Conference on Spectroscopic Ellipsometry (ICSEV).

${ }^{10}$ M. Granata et al., Phys. Rev. D 93, 012007 (2016).

${ }^{11}$ G. M. Harry et al., Classical and Quantum Gravity 24, 405-415 (2007).

${ }^{12}$ R. Bassiri et al., Acta Mater. 61, 1070 - 1077 (2013).

${ }^{13}$ H. B. Callen and R. F. Greene, Phys. Rev. 86, 702 (1952).

${ }^{14}$ Y. Levin, Phys. Rev. D 57, 659-663 (1998).

${ }^{15}$ F. Urbach, Phys. Rev. 92, 1324-1324 (1953).

${ }^{16}$ G. D. Cody et al., Phys. Rev. Lett. 47, 1480-1483 (1981).

${ }^{17}$ P. W. Anderson, Phys. Rev. 109, 1492-1505 (1958).

${ }^{18}$ J. Dong and D. A. Drabold, Phys. Rev. Lett. 80, 1928-1931 (1998).

${ }^{19}$ Y. Pan et al., Phys. Rev. Lett. 100, 206403 (2008).

${ }^{20}$ D. A. Drabold, Eur. Phys. J. B 68, 1-21 (2009).

${ }^{21}$ A. Amato et al., arXiv:1903.06094 (2019).

${ }^{22}$ G. Cagnoli et al., Phys. Lett. A 382, $2165-2173$ (2018).
${ }^{23}$ T. Kessler et al., Nat. Photon. 6, 687-692 (2012).

${ }^{24}$ M. Aspelmeyer et al., Rev. Mod. Phys. 86, 1391-1452 (2014).

${ }^{25}$ A. D. Ludlow et al., Rev. Mod. Phys. 87, 637-701 (2015).

${ }^{26}$ C. Michel et al., Proc. SPIE 8550, Optical Systems Design 8550 , 85501P (2012).

${ }^{27}$ L. Anghinolfi et al., J. Phys. D Appl. Phys. 46, 455301 (2013). ${ }^{28}$ O. Stenzel et al., Appl. Optics 50, C69-C74 (2011).

${ }^{29}$ H. Fujiwara, Spectroscopic ellipsometry: principles and applications (John Wiley \& Sons, 2007).

${ }^{30}$ A. S. Ferlauto et al., J. Appl. Phys. 92, 2424-2436 (2002).

${ }^{31}$ C. M. Herzinger and B. D. Johs, (1998), uS Patent 5,796,983.

${ }^{32}$ J. I. Larruquert and L. V. R. de Marcos, Thin Solid Films 664, $52-59$ (2018).

${ }^{33}$ D. Franta et al., in Optical Characterization of Thin Solid Films, edited by O. Stenzel and M. Ohlídal (Springer International Publishing, Cham, 2018) pp. 31-82.

${ }^{34}$ J. R. Sites et al., Optical Engineering 22, $447-449-3$ (1983).

${ }^{35}$ R. Flaminio et al., Classical and Quantum Gravity 27, 084030 (2010).

${ }^{36}$ A. C. Boccara et al., Opt. Lett. 5, 377-379 (1980).

${ }^{37}$ Y. Pan et al., J Non-Cryst. Solids 354, 3480 - 3485 (2008).

${ }^{38}$ M. Granata et al., Phys. Rev. Materials 2, 053607 (2018).

${ }^{39}$ A. S. Nowick and B. S. Berry, (Academic Press New York, 1972) pp. xv, 677 p.

${ }^{40}$ C. Bundesmann et al., Thin Solid Films 516, $8604-8608$ (2008).

${ }^{41}$ A. Amato et al., J Phys. Conf. Ser. 957, 012006 (2018).

42 N. Mott, Advances in Physics 16, 49-144 (1967).

${ }^{43}$ Y. Aso et al. (The KAGRA Collaboration), Phys. Rev. D 88, 043007 (2013).

${ }^{44}$ F. Travasso et al., Europhys. Lett. 80, 50008 (2007).

${ }^{45}$ E. Cesarini et al., Rev. Sci. Instrum. 80, 053904 (2009).

${ }^{46}$ E. Cesarini et al., Classical and Quantum Gravity 27, 084031 (2010).

${ }^{47}$ M. Granata et al., Arch. Metall. Mater. 60, 365 - 370 (2015).

${ }^{48}$ A. Cadez and A. Abramovici, J. Phys. E Sci. Instrum. 21, 453 (1988). 




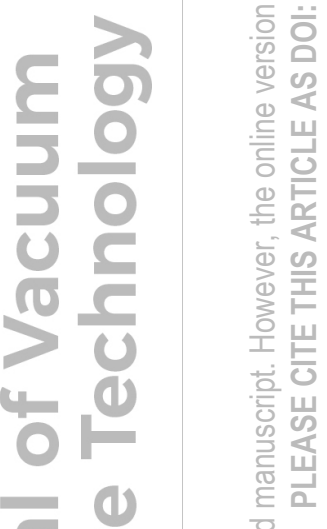

(1) 1

당

(1)

( 1

2

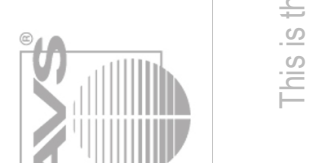




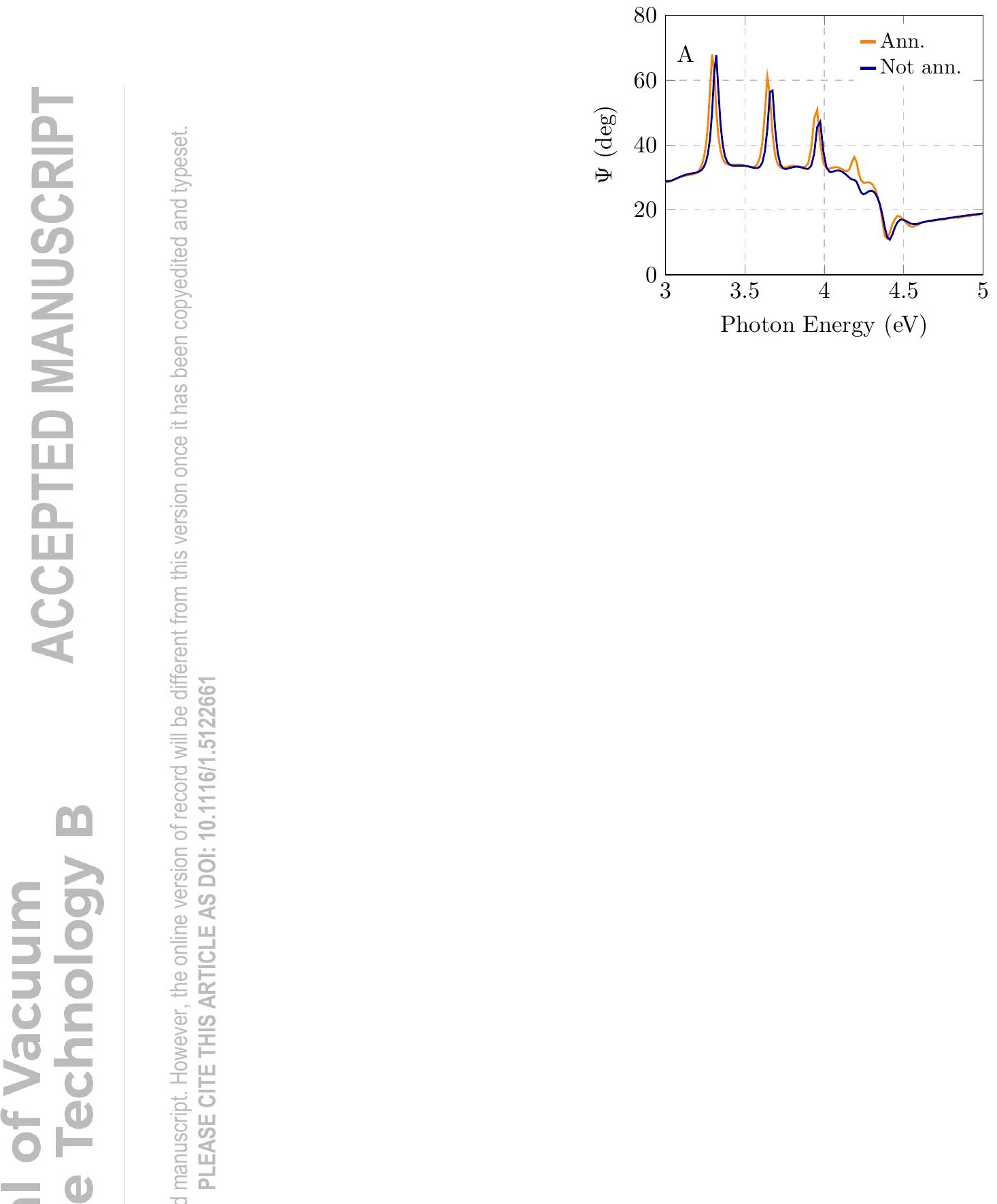

10

b.

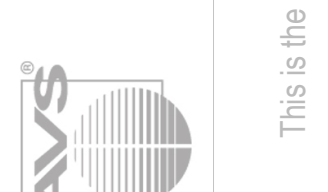





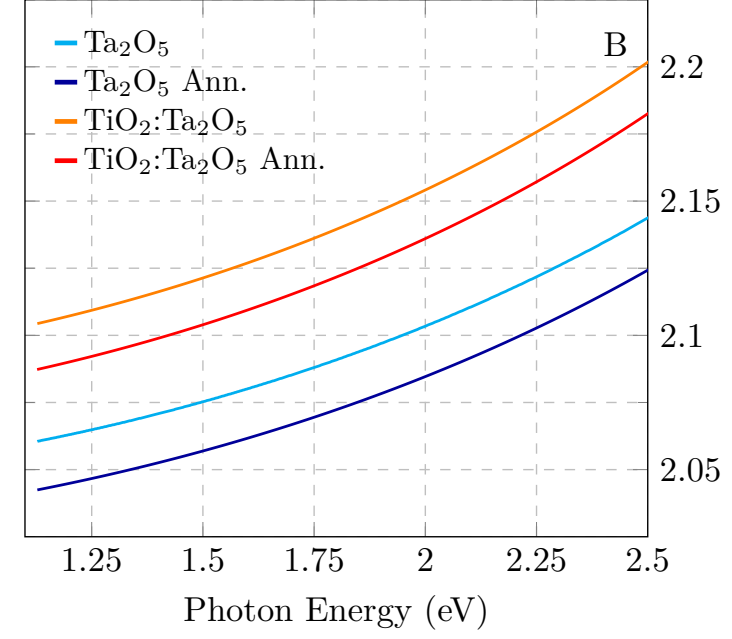




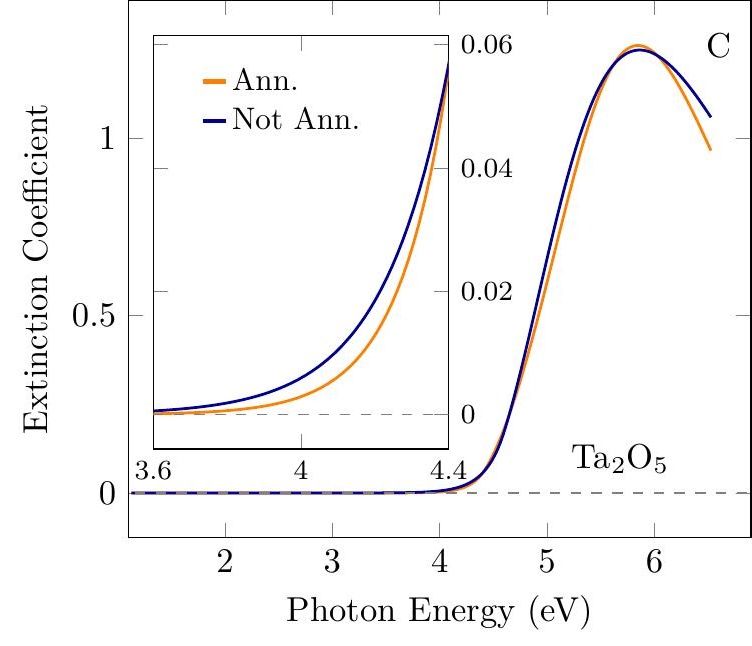

ค 

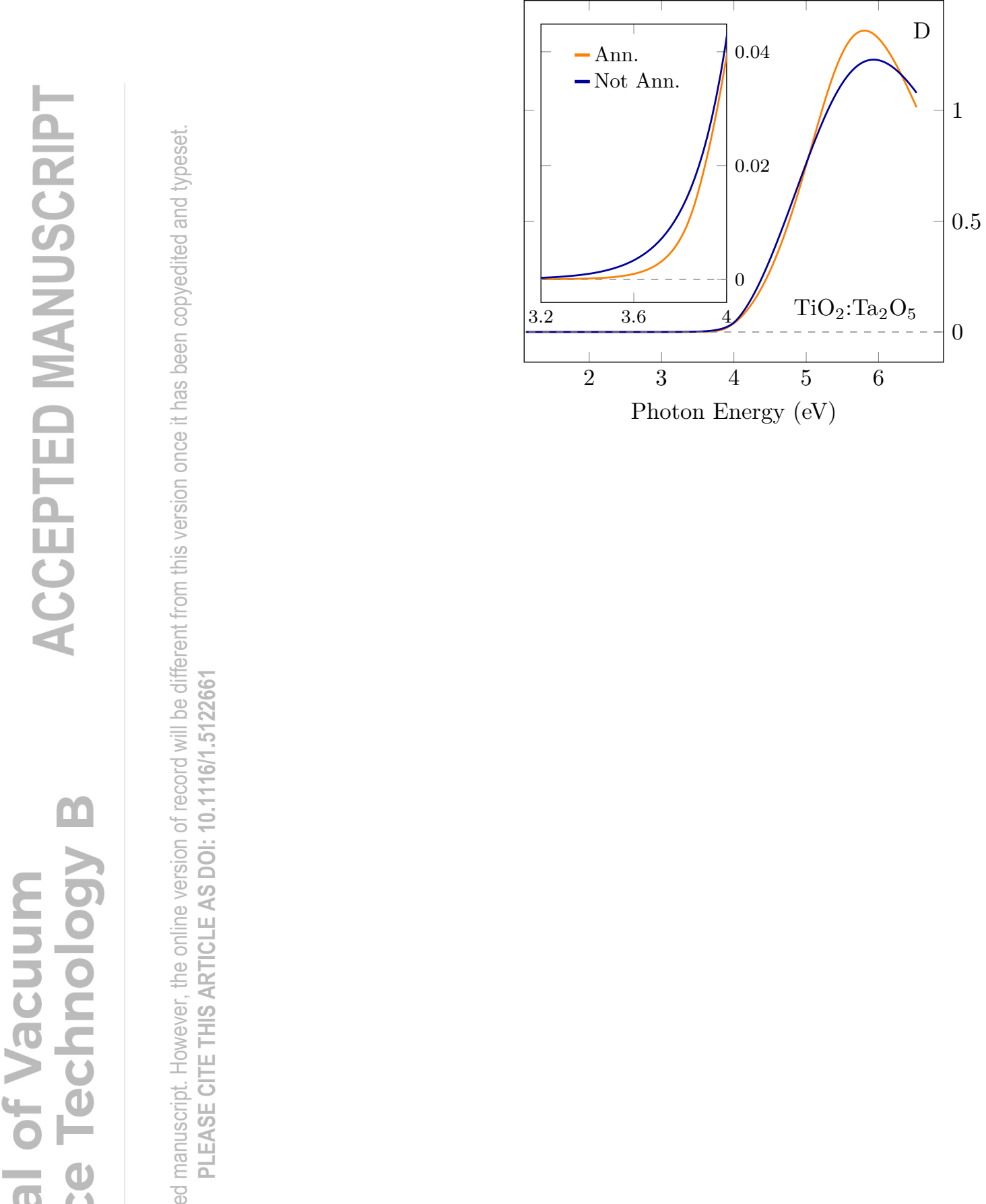

$\begin{array}{ll}10 & 0 \\ 0 & 0 \\ 0 & 0\end{array}$

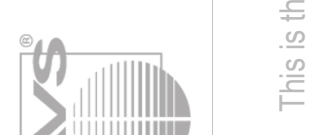

